\title{
Refining associations between TAS2R38 diplotypes and the 6-n-propylthiouracil (PROP) taste test: findings from the Avon Longitudinal Study of Parents and Children Nicholas J Timpson*1,4, Jon Heron², Ian NM Day¹, Susan M Ring1, Linda M Bartoshuk ${ }^{3}$, Jeremy Horwood ${ }^{1}$, Pauline Emmett ${ }^{2}$ and George Davey- Smith ${ }^{1}$
}

Address: ${ }^{1}$ Department of Social Medicine, Bristol University, Bristol, UK, ${ }^{2}$ ALSPAC, Department of Social Medicine, Bristol University, Bristol, UK, ${ }^{3}$ University of Florida, Dept. of Community Dentistry and Behavioral Science, Gainesvile, USA and ${ }^{4}$ The Wellcome Trust Centre for Human Genetics, Oxford University, Oxford, UK

Email: Nicholas J Timpson* - epnjt@well.ox.ac.uk; Jon Heron - Jon.Heron@bristol.ac.uk; Ian NM Day - Ian.Day@bristol.ac.uk; Susan M Ring - S.M.Ring@bristol.ac.uk; Linda M Bartoshuk - lbartoshuk@dental.ufl.edu; Jeremy Horwood - J.Horwood@bristol.ac.uk; Pauline Emmett - P.M.Emmett@bristol.ac.uk; George Davey-Smith - george.davey-smith@bris.ac.uk

* Corresponding author

Published: 28 July 2007

BMC Genetics 2007, 8:5I doi:10.1186/147I-2156-8-5I
Received: I February 2007

Accepted: 28 July 2007

This article is available from: http://www.biomedcentral.com/I47I-2/56/8/5 I

(c) 2007 Timpson et al; licensee BioMed Central Ltd.

This is an Open Access article distributed under the terms of the Creative Commons Attribution License (http://creativecommons.org/licenses/by/2.0), which permits unrestricted use, distribution, and reproduction in any medium, provided the original work is properly cited.

\begin{abstract}
Background: Previous investigations have highlighted the importance of genetic variation in the determination of bitter tasting ability, however have left unaddressed questions as to within group variation in tasting ability or the possibility of genetic prescription of intermediate tasting ability. Our aim was to examine the relationships between bitter tasting ability and variation at the TAS2R38 locus and to assess the role of psychosocial factors in explaining residual, within group, variation in tasting ability.

Results: In a large sample of children from the Avon Longitudinal Study of Parents and Children, we confirmed an association between bitter compound tasting ability and TAS2R38 variation and found evidence of a genetic association with intermediate tasting ability. Antisocial behaviour, social class and depression showed no consistent relationship with the distribution of taste test scores.

Conclusion: Factors which could influence a child's chosen taste score, extra to taste receptor variation, appeared not to show relationships with test score. Observed spread in the distribution of the taste test scores within hypothesised taster groups, is likely to be, or at least in part, due to physiological differentiation regulated by other genetic contributors. Results confirm relationships between genetic variation and bitter compound tasting ability in a large sample, and suggest that TAS2R38 variation may also be associated with intermediate tasting ability.
\end{abstract}

\section{Background}

Variation in the ability to taste PTC, PROP and related compounds has been recognised as one of the classical markers of population genetics. Since the seminal find- ings of Blakeslee and Fox $[1,2]$, the distribution of PTC/ PROP tasting has been extensively analysed. This variation has been noted to vary from the extremes of "taste blindness" (a lack of sensitivity, or non-taster of PTC/ 
PROP), to apparent "super tasting" (an extra sensitive reaction to the bitterness of PTC/PROP) and has shown marked variation in the distribution of such traits across populations (taste blindness ranging from 3\% in West Africa, to $6-23 \%$ in China, $40 \%$ in India and around 30\% in North American Caucasian populations [3,4]).

Conventional assessment of bitter tasting ability has been by PTC (phenylthiocarbamide)/PROP taste challenge and response assessment. It was found that all bitter compounds containing the thiocyanate $(\mathrm{N}-\mathrm{C}=\mathrm{S})$ moiety elicit bimodal patterns of response [5]. In addition to this it has been shown that PTC taste responses are strongly correlated with all of these compounds [6], as is the case for PROP [3].

Initial genetic analyses presented indications of bitter compound tasting ability as a complex genetic trait, but provided little specific evidence as to possible causal, or robustly associated genetic components [4,7-11]. However, specific positional cloning efforts have since identified a region of chromosome 7 (in the TAS2R38 gene), which has shown patterns of haplotypic association which are associated with specific measurements of bitter tasting ability [12]. This has provided direct evidence of a physiological link between genetic variation and tasting ability and has prompted hypotheses as to the possible relevance of bitter tasting for ultimate diet choice and related health [13-15]. We note that the human genome project has now identified more than twenty genes for bitter taste [16], likely to also associate with health effects.

Existing studies assessing relationships between TAS2R38 haplotype variation and specific bitter tasting ability have limited samples sizes and have largely been restricted to the assessment of relationships between genetic variation at the TAS2R38 locus and the binary measure "taster/nontaster". Whilst there is evidence as to the existence of this association, there has not to date, been large-scale replication or refinement of these observations. Furthermore, whilst diplotypes of the TAS2R38 gene locus are thought to prescribe one's ability to detect the bitter compounds PROP and PTC (PROP representing a suboptimal, but effective ligand for the TAS2R38 receptor), little its known about the effects/association of haplotypes at intermediate/lower frequency or the cause of the distribution of bitter tasting ability within haplotype defined groups.

Studies into the psychophysiology of PROP detection have suggested that in addition to tasters and non-tasters, there also "supertasters" of bitter compounds. Supertasters of PROP are believed to be tasters who also have unusual tongue anatomy: a high density of fungiform papillae, the structures that house taste buds. Supertasting is not linked to variation et the TAS2R38 locus [15].
The present analyses are focussed on "taster" or "nontaster" as the only previous grouping of note related to TAS2R38 locus variation. It is therefore not the existence of super tasting that is in question, more the refinement of the currently crude relationships recorded between genetic variation at the TAS2R38 locus and the ability to taste specific bitter compounds. It is predicted, that with a study of this magnitude, the observation of the rare AA haplotype at the TAS2R38 locus within the ALSPAC cohort will allow investigation of its potential link to intermediate tasting ability [17].

Such an investigation, together with the suggestion of subjectivity in the rating of PROP taste test scores [18], also raises the question as to the determination of the distribution of PROP taste test scores within genetically prescribed tasting groups. Whilst physiological factors such as the number of taste buds may be instrumental in the determination of this variation [19], it has been suggested that psychological/behavioural factors play some role [20].

Here we analyse PROP bitter taste test scores collected in the ALSPAC child cohort and their relation to known variation at the TAS2R38 locus. Also, the availability of a series of psychological/behavioural measures reflecting factors thought to influence the ultimate response of a child to this taste challenge, has allowed the investigation of residual variation in bitter tasting ability. As such, we aim to comment on the possible origins of the distribution of taste scores found within the prior defined haplotypic taste groups.

\section{Results}

Of the 13988 cases/pregnancies in the ALSPAC cohort, samples from $\mathrm{n}=9765$ children and $\mathrm{n}=8736$ mothers formed a working population of $\mathrm{n}=12234$ unrelated individuals (all genotyped children and unrelated mothers where children were not available, comprising $83 \%$ of the cohort) available for haplotypic reconstruction. Of this group, $\mathrm{n}=8$ had one missing genotype and $\mathrm{n}=174$ had missing data for two genotypes. Genotyping of the TAS2R38 variants $\mathrm{P} 49 \mathrm{~A}$ and $\mathrm{A} 262 \mathrm{~V}$ yielded minor allele frequencies of 0.40 and 0.45 respectively, Table 2. From these genotyping results, haplotypic reconstruction resolved the common haplotypes $\mathrm{AV}$ and $\mathrm{PA}$ at frequencies of 0.55 (SE 0.0004) and 0.40(SE 0.0004) within this population, Table 3 . The rarer haplotypes AA and PV were observed at frequencies 0.05 (SE 0.0003) and 0.001(SE 0.00008).

$\mathrm{n}=4795$ children completed a PROP taste test analysis and were carried forward into further analysis. Figure 1 shows the distribution of PROP taste test scores in the ALSPAC population. From the 168 children who completed the PROP taste test twice, the correlation of scores 
Table 2: Allele frequencies at variant loci TAS2R38 P49A and A262V in the ALSPAC sample

\begin{tabular}{|c|c|c|c|c|c|c|}
\hline Nucleotide & Amino Acid & Allele & Protein code & Frequency in current study & $\mathrm{p}^{*}$ & Comparative frequencies (Kim et al 2003) \\
\hline \multirow[t]{2}{*}{145} & 49 & G & Ala & $0.60(n=10977)$ & 0.9 & 0.64 \\
\hline & & $\mathrm{C}$ & Pro & $0.40(n=7467)$ & & 0.36 \\
\hline \multirow[t]{2}{*}{262} & 262 & $\mathrm{~T}$ & Val & $0.55(n=10147)$ & 0.7 & 0.62 \\
\hline & & C & Ala & $0.45(\mathrm{n}=8405)$ & & 0.38 \\
\hline
\end{tabular}

* indicates $\mathrm{p}$ value for test of Hardy Weinberg Equilibrium (exact test [37])

between both tests was $0.62\left(r^{2}=0.39\right)$. This relationship yielded a regression coefficient of $0.61(0.49,0.72) . \mathrm{p}=<$ 0.001 . Figure 2 shows the distribution of differences that exist between taste test scores for the 168 children over these two time points. The mean time between the two measurements for this group was 33.4 days.

The combination of PROP taste test data with haplotypic information yielded a test population of $\mathrm{n}=4178$ children. Of note, in analysis of PROP taste test scores by PC haplotype, none of the reconstructed haplotypic values fell below the cut off posterior reconstruction threshold probability of 0.8 .

The distribution of PROP taste test scores by haplotypic combination (diplotype) is shown in Table 4. PROP taste test score was observed to be lowest for the haplotypically predicted non-tasters (AV/AV - median 3.72, IQR [5.2]), whilst predicted tasters had high PROP taste test scores with the highest being for homozygous taster haplotypes (PA/PA - median 8.07, IQR [2.5]). Of note, whilst the carriage of the PA haplotype appeared to confer tasting ability throughout, the presence of the rarer heterozygote AV/ AA showed evidence for intermediate tasting ability.

Pairwise differences in mean PROP taste test score showed expected patterns according to diplotype comparison (Table 5). The largest difference in tasting response was seen between diplotypes AV/AV (non-taster) and PA/PA (homozygote taster), (4.35 95\% CI [4.13, 4.56]). In contrast, the smallest difference between diplotypes AV/PA (heterozygote taster) and AA/PA (heterozygote taster, $(0.1095 \%$ CI $[-0.26,0.46])$. The presence of the rarer hap- lotype AA (when in comparison to either taster or nontaster diplotypes) exhibited moderate pairwise differences (e.g. AV/AA versus AV/AV pairwise difference of $1.6595 \%$ CI $[1.21,2.10]$ and AV/AA versus PA/PA pairwise difference of $2.70,95 \%$ CI $[2.26,3.13])$.

From these observations, the generation of predicted tasting ability groups was permitted and allowed the analysis of the distribution taste scores between them, Figure 3 . Clear differences in the distribution of PROP taste test scores were observed between the haplotypically prescribed tasting groups, as confirmed by a rank sum test for differences between these groups $(\mathrm{z}=-35.302, \mathrm{p}=<$ $0.001)$. Furthermore, the creation of a third group defined by the carriage of the rare haplotype AA (Figure 4), whilst only observed in relatively small numbers $(n=207$ excluding those with the common PA taster haplotype on the basis of apparently dominant effects) revealed patterns indicative of intermediate tasting ability when compared to the main group distributions, this was again confirmed by a rank sum test for differences between these groups $(\mathrm{z}=-7.132, \mathrm{p}=<0.001)$ (Figure 2).

Analysis of quartiles of taste test score with respect to their relationship with factors which could potentially explain the spread of PROP taste test results within the haplotype defined test groupings seen in Figure 3., showed no strong evidence of trend effect. When assessed in both homozygote taster predicted individuals and homozygote nontaster predicted individuals (in efforts to avoid noise from differing genetic background), social class, depression and antisocial behaviour (thought to be potentially influencing child's reporting of taste sensation despite physiologi-

Table 3: Haplotype frequencies at variant loci TAS2R38 P49A and A262V in the ALSPAC sample

\begin{tabular}{ccccc}
\hline Haplotype & P49A & A262V & Observed Frequency & Comparative frequencies (Kim et al 2003) \\
\hline AV & G & T & 0.546 & 0.47 \\
AA & G & C & 0.047 & - \\
PA & C & C & 0.406 & 0.49
\end{tabular}

Haplotype frequencies (common haplotypes representing $\sim 99 \%$ of all variation) are derived from a sample base $n=12234$ (derived from $n=9399$ children, $n=2661$ mothers where child has no genotype data and 174 missing data points). Haplotypes for P49A/A262V genotypes obey Hardy Weinberg Equilibrium ( $P=0.8$, chitesti [38]). The rare haplotype PV was observed in the ALSPAC population at a frequency of $0.00 \mathrm{I}$. 


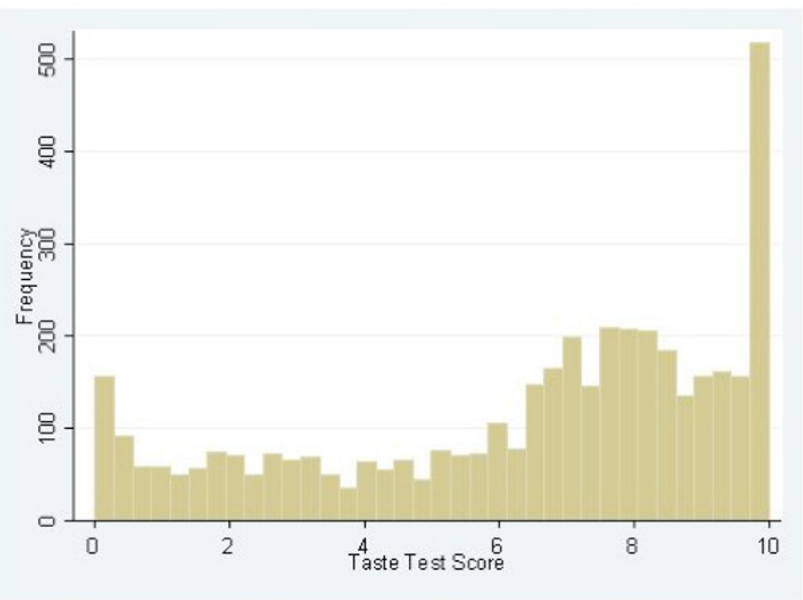

Figure I

Distribution of PROP taste scores in the ALSPAC cohort by frequency. Taste test score derived from a general labelled magnitude scale.

cal response) appeared to show no consistent relationship with the spread of taste test results, tables 6 and 7 .

\section{Discussion and conclusion}

As a study containing data on $\mathrm{n}=4178$ PROP taste test scores and concurrent TAS2R38 haplotypes, analysis in the ALSPAC cohort is the largest study to date to address the question of the haplotypic association with tasting ability. This is the first instance of the application of the gVAS to children in this manner and has shown this to be a consistent approach to the measurement of tasting ability. Resolution of common haplotypes across the TAS2R38 locus revealed variants adhering to Hardy Weinberg Equilibrium and for which frequencies were close to those reported elsewhere [12,21].

Analysis of the PROP taste test scores in the ALSPAC cohort has replicated previous findings of association between variation at the TAS2R38 locus and response bitter taste test challenge. As predicted by the findings of Kim et al [12], the common PA haplotype predicted those able to taste the bitter compound PROP when at least one copy was carried. Alternatively, homozygotes of the other common haplotype, AV, (haplotypes AV and PA accounting for $>90 \%$ of $2 \mathrm{~N}$ ) exhibited clear patterns of taste insensitivity to the bitter PROP compound.

Importantly, the predicted intermediate tasting ability of those carrying the rare AA haplotype [17] (in cases excluding heterozygotes with one copy of the common and "taster" defining PA haplotype) appears to be demonstrated in the ALSPAC cohort.

As yet unreported, we were also interested in the presence and patterning of variation in taste test scores within the genetically prescribed tasting groups for PTC/PROP. We performed an investigation into the distribution of PROP taste test scores within genetically homogeneous groups of tasters (homozygote PA individuals) and non-tasters (homozygote AV individuals). This revealed that three select factors (depression, social class and antisocial behaviour) thought to influence the response of children to bitter taste challenge did not appear to be associated with the variation in taste test score in these groups.

On the basis of current observations, it is suggested that physiological differentiation, likely derived from further genetic factors $[8,9,11,22]$, may form the basis of variation in taste score within groups previously defined as "tasters/nontasters" by TAS2R38 haplotypes. These further possible physiological factors include not only alternative taste receptor complexes, but possibly factors previously associated with the existence of "super tasting" ability [23-26]. Importantly, this notes that (i) residual variation in this particular taste test score, after previously recognised haplotype groupings, is likely to be due to other physiological mechanisms and (ii) that there may be a degree of phenotypic continuity in this trait recognised classically as bimodal in character. In addition to this, we observed that in the overall population, the classification of all individuals into the broad categories "taster/nontaster" may not be appropriate. Evidence from

Table 4: Median PROP taste test scores (on a scale of 0-10 with 10 being the most intense taste response) by diplotype in the ALSPAC cohort

\begin{tabular}{ccccc}
\hline Diplotype & $\mathrm{N}$ & Predicted PROP tasting ability & Median PROP Taste Score \\
\hline AV/AV & 1202 & Non-taster & 3.72 & 5.37 \\
AV/AA & 202 & (Intermediate) & 7.48 & 5 \\
AV/PA & 1893 & Taster & 7.58 & 2.6 \\
AA/PA & 140 & Taster & 8.07 & 2.85 \\
PA/PA & 727 & Taster & 2.5 \\
\hline
\end{tabular}

Common haplotypic combinations shown above account for $>99 \%$ of the sample population included in analysis. IQR represents interquartile range.

Derived from a sample base of 4178 (i.e. all those remaining with PROP taste test scores post haplotypic exclusion). 


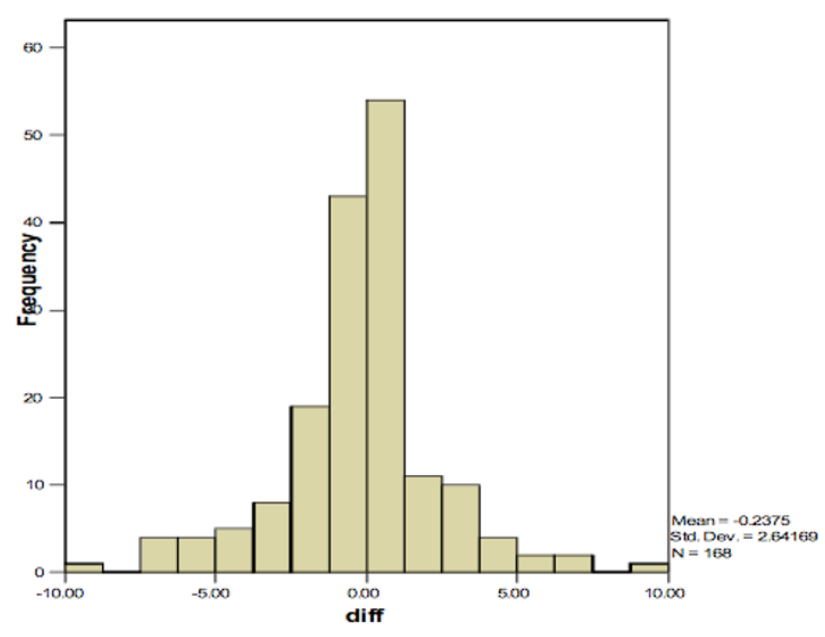

Figure 2

Distribution of differences between multiple taste test assessments in the ALSPAC cohort. Distribution of differences observed between general visual analogue scale scores for the description PROP taste test results within 168 repeat tested children from the ALSPAC cohort. Mean difference between two measures is $0.24(0.16,0.64)$, $P$ $=0.25$.

this study suggests that the carriage of particular haplotypic combinations is associated with intermediate bitter compound tasting ability.

\section{Methods}

The Avon Longitudinal Study of Parents and Children (ALSPAC) is a geographically based cohort that recruited pregnant women residing in Avon with an expected delivery date between $1^{\text {st }}$ April 1991 and 31 $1^{\text {st }}$ December 1992. 14541 pregnant women were initially enrolled, with 14 062 children born. This represents $80-90 \%$ of the eligible population $[27,28]$. Of these children, 13988 were alive at 12 months. From this, two sampling frames were to be adhered to (i) the maximal population sample for haplo- typic reconstruction (including children with DNA samples and mothers with DNA samples where a child's DNA sample was missing) and (ii) the analysis data set including just children with at least one genotype and concurrent PROP taste test results. This formed a maximal baseline population of $\mathrm{n}=12371$ (reduced to $\mathrm{n}=12234$ with the loss of one sib from twin pairs) for which, in order to allow investigation, ethical approval was obtained from the ALSPAC Law and Ethics Committee, and local research ethics committees.

\section{PROP test}

Paper disks impregnated with PROP have been shown to be a crude but rapid way to test responses to PROP in large groups [19]. As part of a face-to-face clinic session held when the children were aged 10 years, a nutritionist interviewed them about their diet. Following this, the nutritionist proceeded to assess the subject's reaction to a bitter (PROP) challenge using a general visual analogue scale (gVAS) [29].

The disks were prepared by soaking circular pieces of filter paper (Whatman \#1) in a saturated solution of PROP (at near boiling temperature) and then drying them. The PROP crystallizes into the paper thus allowing the paper to serve as a convenient way to permit a subject to taste a limited quantify of PROP crystals. The PROP crystals go into solution in the subject's saliva and produce a high concentration of PROP at the taste receptor sites. The paper produces bitterness approximately equivalent to a solution of $.0032 \mathrm{M}$, close to the highest concentration of PROP that will remain in solution when PROP solutions are refrigerated for storage. The purpose of using a high concentration for screening is that PROP functions for nontasters, medium tasters and supertasters diverge; thus the highest practical concentration of PROP produces the most accurate sorting.

In this test, the nutritionist explained to the child that they were going to taste a piece of paper and would then mark

Table 6: Behavioural variables (number (\%)) by quartile of PROP taste score for AVIAV homozygotes (non-tasters).

\begin{tabular}{|c|c|c|c|}
\hline Homozygote non-taster (AV/AV) & Manual social class $(n=1055)$ & Antisocial activities $(\mathrm{n}=1190)$ & Depressed tendency $(n=1174)$ \\
\hline \multirow[t]{2}{*}{1} & 129 & 55 & 83 \\
\hline & $(48.86)$ & $(18.46)$ & $(28.04)$ \\
\hline \multirow[t]{2}{*}{2} & 130 & 26 & 75 \\
\hline & $(47.79)$ & $(8.61)$ & $(25.34)$ \\
\hline \multirow[t]{2}{*}{3} & 119 & 49 & 68 \\
\hline & $(45.25)$ & $(16.23)$ & $(23.05)$ \\
\hline \multirow[t]{2}{*}{4} & 116 & 42 & 88 \\
\hline & $(45.31)$ & $(14.58)$ & $(30.66)$ \\
\hline linear test, $P$ & 0.3 & 0.7 & 0.01 \\
\hline
\end{tabular}

Note, reference groups are not shown. I, 2, 3, 4 represent grouping by quartiles of PROP taste test (general labelled magnitude scale). 
Table 7: Behavioural variables (number (\%)) by quartile of PROP taste score for PA/PA homozygotes (tasters).

\begin{tabular}{|c|c|c|c|}
\hline Homozygote taster (PA/PA) & Manual social class $(n=646)$ & Antisocial activities $(n=72 I)$ & Depressed tendency $(n=710)$ \\
\hline \multirow[t]{2}{*}{1} & 69 & 29 & 38 \\
\hline & $(42.07)$ & $(15.85)$ & $(21.47)$ \\
\hline \multirow[t]{2}{*}{2} & 65 & 31 & 32 \\
\hline & $(36.93)$ & $(15.82)$ & $(16.58)$ \\
\hline \multirow[t]{2}{*}{3} & 71 & 23 & 40 \\
\hline & $(46.10)$ & $(13.37)$ & (23.39) \\
\hline \multirow[t]{2}{*}{4} & 71 & 32 & 53 \\
\hline & $(46.7 I)$ & $(18.82)$ & $(31.36)$ \\
\hline linear test, $P$ & 0.2 & 0.6 & 0.6 \\
\hline
\end{tabular}

Note, reference groups are not shown. I, 2, 3, 4 represent grouping by quartiles of PROP taste test (general labelled magnitude scale).

how strong they thought the sensation of the taste was on a line. The child was asked to name their senses and was prompted if he/she could not. The nutritionist then asked the child to describe the loudest, most intense sound they had ever heard and the brightest, most intense light they had ever seen. They then pointed to the scale on the datasheet (a $10 \mathrm{~cm}$ line), explaining that the left end of the scale represented no sensation and the right end represented the most intense sensation, explaining again the most intense sensation as the loudest sound or brightest light that they had just described. The child was then asked to point on the line where a whisper and where a shout would go on the scale. If children understood the instructions, a whisper would be rated as less intense than a shout which, in turn, would be rated less intense that the loudest sound. Once children rated the sounds in the correct order, they were asked to place a disc of PROP paper on their tongue and move it around for about 10 seconds. The child was then given a pen and asked to make a mark on the line. Once the child had left the room the nutritionist measured how far from the left the child had marked (10 $\mathrm{cm}$ being the maximum).

Of note, the instructions regarding the whisper were used to check that the child understood how the scale worked and which end represented the largest magnitude of stimulation in regard to the senses.

\section{PROP taste test consistency}

Within the focus at 10 years of age follow-up screens, a small subset of the ALSPAC cohort who under took taste tests were re-invited to perform the test again (i.e. a repeat of the clinic at 10 years). Of 237 children assigned re-invitation (approximately 3\% of the total sample set) 203 children completed the test the first time and 196 the second time. In total, 168 children performed the test twice. These provided data for the assessment of internal consistency for the PROP taste test. Consistency was assessed by analysis of the correlation between test scores at both measurements and by the regression of repeat test data.

\section{Genotyping}

Genetic variants were selected on the basis of previous literature indicating their association with PTC taste test scores. SNPs to be investigated here are the A49P and V262A variants of the TAS2R38 gene on Ch7 (rs713598 and rs1726866 respectively). Both of these are non-synonymous protein change causing variants the former leading to a Proline/Alanine change at amino acid position 145, whilst the latter leads to a Alanine/Valine change at amino acid position 785 . As such, these are taken as likely to be functionally significant in relation to the action of the TAS2R38 gene (although the actual implication of these variants are unknown) [12].

DNA, from cord blood or peripheral blood was extracted and processed as described previously [30]. SNPs were genotyped using the KASPar chemistry which is a competitive allele specific PCR SNP genotyping system using FRET quencher cassette oligos[31]. All genotyping was performed by KBioscience [32].

\section{Psychosocial factors}

Social class was derived using OCPS classifications [33] with information taken from a self completed questionnaire administered to the mothers at 32 weeks of pregnancy. This information yielded a 6-level variable I, II, III non-manual, III manual IV, V, which was dichotomised centrally for a manual/non-manual split.

For the assessment of depression the children were given a series of envelopes with statements written on them about how they might have been feeling or acting in the previous two weeks. This was done at the same clinic session. Depression was assigned in cases where 6 or more indicators of depression existed. This represented the top quartile of the population distribution. The statements used have been taken from the Short Mood and Feelings Questionnaire [34], which has been designed to provide a rapidly administered questionnaire for use in epidemiological studies. 
Table 5: Pairwise differences of mean PROP taste test score by diplotype in ALSPAC

\begin{tabular}{|c|c|c|c|c|c|}
\hline$*$ & AVIAV & AVIAA & AV/PA & AA/PA & PA/PA \\
\hline AVIAV & $*$ & Non-taster Intermediate & Non-taster Taster (Het) & Non-taster Taster (Het) & Non-taster Taster (Hom) \\
\hline AVIAA & $1.65(1.21,2.10)$ & $*$ & Intermediate Taster (Het) & Intermediate Taster (Het) & Intermediate Taster (Hom) \\
\hline AV/PA & $3.76(3.57,3.95)$ & $2.11(1.68,2.53)$ & $*$ & Taster (Het) Taster (Het) & Taster (Het) Taster (Hom) \\
\hline AA/PA & $3.86(3.48,4.24)$ & $2.10(1.67,2.75)$ & $0.10(-0.26,0.46)$ & $*$ & Taster (Het) Taster (Hom) \\
\hline PA/PA & $4.35(4.13,4.56)$ & $2.70(2.26,3.13)$ & $0.59(0.42,0.76)$ & $0.49(0.11,0.86)$ & $*$ \\
\hline
\end{tabular}

Pairwise difference of mean PROP taste score displayed with predicted tasting status in opposing matrix space. Estimates and $95 \%$ Cl derived from categorical regression of taste test score by diplotype with robust SE.

Lastly, antisocial behaviour was defined by the presence of any 1 of 11 antisocial activities report by the child during the same clinic session, covering a range of behaviours including stealing, cruelty to animals, smoking, drinking, taking drugs and a series of dummy questions. Questions were derived from the self-reported antisocial behaviour for young children questionnaire [35]. A variable was then derived indicating whether any such activity had been carried out.

For the purpose of analysis of the distribution of taste test scores by social class, antisocial behaviour and depression, tasting scores were organised into quartiles.

\section{Statistical analysis}

Table 1. summarises haplotypes and predicted taster status identified in relation to previous literature (i.e. an individual's status as either a 'taster' or 'non-taster' of bit-

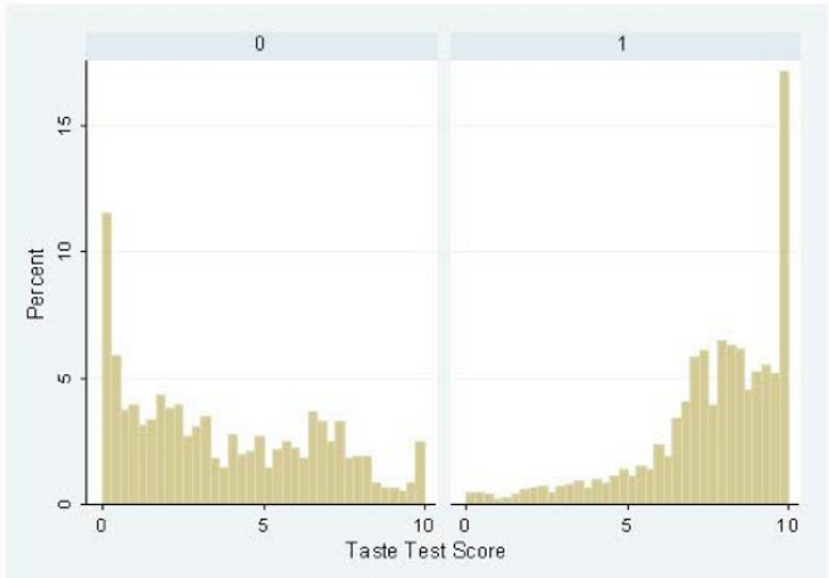

Figure 3

Distribution of PROP taste test scores by genetic prediction of tasting ability in the ALSPAC cohort. Test groups here correspond to $0=$ non-tasters as defined by the homozygous carriage of the TAS2R38 haplotype AV; I $=$ tasters as defined by the homozygous or heterozygous carriage of the TAS2R38 haplotype PA. Ranksum analysis for comparison of random points from these two distributions yields $\mathrm{P}=<0.00 \mathrm{I}$. ter compounds). Haplotype names 'PAV' and 'AVI' refer to recognised 'taster' and 'non-taster' states respectively [12] and are specifically derived from their protein coding sequences. In the context of this study, haplotypes 'AVI' and 'PAV' have been renamed AV and PA respectively. An individual was therefore designated a 'non-taster' if they carried two copies of AV and a 'taster' if they carried at least one copy of PA.

Previous results [12] demonstrated the existence of the variant site TAS2R38 V296I (34 bases from TAS2R38 $\mathrm{V} 262 \mathrm{~A}$ ) in total linkage disequilibrium (LD) with TAS2R38 V262A in a European population. In light of this, it was felt appropriate that the genotyping of TAS2R38 P49A and TAS2R38 A262V alone would allow the effective tagging of common haplotypes in this region.

Having collected genotype data, haplotypes were constructed using the programme PHASE (version 2.02, [36],

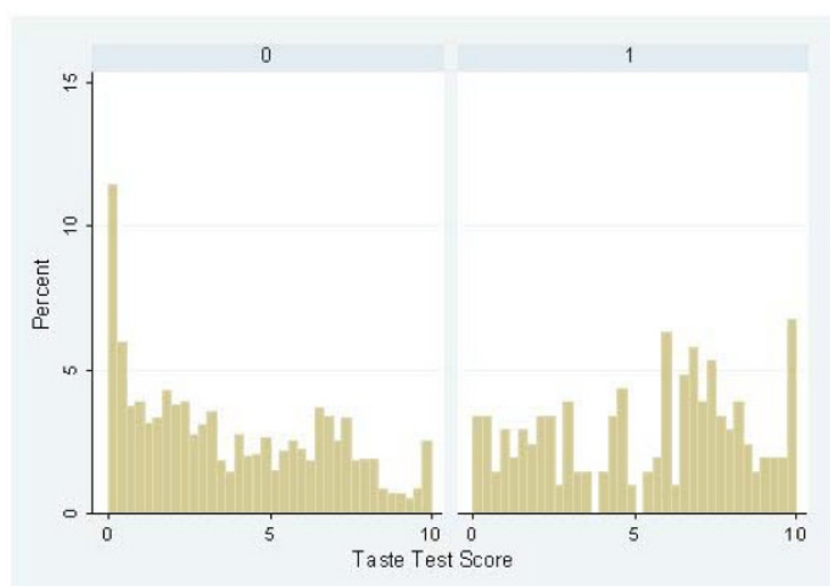

Figure 4

Disrtibution of PROP taste test scores by the rare AA haplotype in the ALSPAC cohort. Test groups here correspond to $\mathrm{I}=$ any carriage of the rare AA haplotype, excluding carriers of the common PA taster haplotype, i.e. predicted intermediate tasters; $0=$ all other individuals. Ranksum analysis for comparison of random points from these two distributions yields $p=<0.00 \mathrm{I}$. 
Table I: Prediction of tasting ability by TAS2R38 haplotype

\begin{tabular}{cccccc}
\hline Kim et al haplotype & Present Study Label & P49A rs713598 & A262V rsl726866 & {$[\mathrm{V} 2961]$} & Taster Status \\
\hline AV [I] & AV & G & T & {$[\mathrm{A}]$} & Non-taster if homozygote \\
AA [V] & AA & G & C & {$[\mathrm{G}]$} & Small effects on non-taster status when heterozygote \\
& & & & AVI/AAV \\
PV [I] & PV & C & T & {$[A]$} & unknown \\
PA [V] & PA & C & C & {$[\mathrm{G}]$} & Taster if homozygote/heterozygote \\
\hline
\end{tabular}

(Haplotypes taken from Kim et al [8]. Haplotype labels are derived from amino acid coding patterns. Square brackets indicate exclusion of variant in present study due to linkage disequilibrium with included variants.)

http://www.stat.washington.edu/stephens/phase.html). This software employs a Bayesian method for the reconstruction of chromosomal phase using genotype data and generates counts and frequencies of observed haplotypes. The underlying method in this approach is a Markov Chain-Monte Carlo procedure in which the probability of preceding observations (in this case unambiguous phase information) allows population genetic inference about unresolved haplotypic phase. Having run phase, posterior probabilities of phase reconstruction were employed to allow the incorporation of a haplotype accuracy cut off for further analysis. For the purpose of taste test analysis, this was constrained at $80 \%$ accuracy for the reconstruction of PTC haplotypes.

Initial analysis considered median (due to skewed distribution of PROP taste test scores) PROP taste test scores by the haplotypic combination (diplotype) carried by those in the ALSPAC sample. Based on Kim and colleagues [12], our prior hypothesis was that bitter tasting ability would differ according to taster status as defined by haplotypic complement (homozygous $\mathrm{AV}=$ non-taster, heterozygous or homozygous PA = taster, other $=$ excluded). This model formed the basis of test groups in taste test scores were included for analysis. To assess the distribution of PROP taste test results for the rarer AA haplotype, a further grouping was generated as defined by any carriage of the AA haplotype (excluding the common and taster predicting PA haplotype) versus the rest of the sample. This grouping was largely designed on the basis of the findings of Bufe et al [17] and was deigned to assess the potentially intermediate tasting ability of those carrying the rare AA haplotype.

Due to the expected bimodal distribution of PROP taste test results, the non-parametric rank sum approach to testing for differences between the properties of test groups was used in this analysis. This also prompted the use of robust standard errors in the presentation of mean pairwise differences in taste test score by diplotypes along with their 95\% CI. Test statistics for the analysis of quartiles of PROP taste test with social class, depression and antisocial behaviour were generated by via simple trend analysis.
All analyses were performed in STATA version 8.2.9 and SPSS version 9.12 .

\section{Competing interests}

The author(s) declare that they have no competing interests.

\section{Authors' contributions}

NT performed the statistical analysis and coordinated the writing of the paper and acts as guarantor

$\mathrm{JH}$ sourcing and piloting of materials and assisted in project management of data collection

INMD inputs to improvements phase of analyses and manuscript

SR production of DNA bank and overseeing provision of DNA and linking genetic data

LB was involved in original data collection and contributed to paper revision

PE - lead the team that administered the PROP challenge having taken advice from LMB on the correct procedure, she was fully involved in the writing of the manuscript.

GDS analysis plan and revising manuscript

\section{Acknowledgements}

We are extremely grateful to all the families who took part in this study, the midwives for their help in recruiting them, and the whole ALSPAC team, which includes interviewers, computer and laboratory technicians, clerical workers, research scientists, volunteers, managers, receptionists, nurses and particularly the nutrition team who administered the PROP challenge. The UK Medical Research Council, the Wellcome Trust and the University of Bristol provide core support for ALSPAC. This publication is the work of the authors who also serve as guarantors for the contents of this paper. NT is funded by a MRC studentship. JH is funded by MRC/Wellcome. INMD group is funded by UK MRC, BHF, and University of Bristol. LB is funded by the National Institute of Deafness and other Communicative Disorders (NIDCD); from grant DC 000283.

\section{References}

I. Blakeslee AF, Fox AL: Our different taste worlds. Journal of Heredity 1932, 23:97-108. 
2. Fox $A L$ : The relationship between chemical constitution and taste. Proceedings of the National Academy of Sciences 1932, 18:115-120.

3. Tepper BJ: 6-n-Propylthiouracil: a genetic marker for taste, with implications for food preference and dietary habits. American Journal of Human Genetics 1998, 63(5): I 27|- 1276.

4. Guo SW, Reed DR: The genetics of phenylthiocarbamide perception. Annals of Human Biology 200 I, 28(2): I I I-I 42 .

5. Harris $\mathrm{H}$, Kalmus $\mathrm{H}$ : Chemical specificity in genetical differences of taste sensitivity. Ann Eugenics London 1949, I 5(I):32-45.

6. Barnicot NA, Harris $H$, Kalmus $H$ : Taste thresholds of further eighteen compounds and their correlation with P.T.C. thresholds. Ann Eugenics london 1951, 16(2): 119-128.

7. Conneally PM, Dumont-Driscoll M, Huntzinger RS, Nance WE, Jackson CE: Linkage relations of the loci for Kell and phenylthiocarbamide taste sensitivity. Human Heredity 1976, 26(4):267-27I.

8. Olson JM, Boehnke M, Neiswanger K, Roche AF, Siervogel RM: Alternative genetic models for the inheritance of the phenylthiocarbamide taste deficiency. Genetic Epidemiology 1989, 6(3):423-434

9. Reddy BM, Rao DC: Phenylthiocarbamide taste sensitivity revisited: complete sorting test supports residual family resemblance. Genetic Epidemiology 1989, 6(3):413-42I.

10. Reed DR, Nanthakumar E, North M, Bell C, Bartoshuk LM, Price RA Localization of a gene for bitter-taste perception to human chromosome 5pI5. American Journal of Human Genetics 1999, 64(5): $1478-1480$.

II. Drayna D, Coon H, Kim UK, Elsner T, Cromer K, Otterud B, Baird L, Peiffer AP, Leppert M, Utah Genetic Reference P: Genetic analysis of a complex trait in the Utah Genetic Reference Project: a major locus for PTC taste ability on chromosome 7q and a secondary locus on chromosome 16p. Human Genetics 2003, I I 2(5-6):567-572.

12. Kim UK, Jorgenson E, Coon H, Leppert M, Risch N, Drayna D: Positional cloning of the human quantitative trait locus underlying taste sensitivity to phenylthiocarbamide. Science 2003, 299(56I0): $1221-1225$.

13. Duffy VB, Lucchina LA, Bartoshuk LM: Genetic Variation in Taste: Potential Biomarker for Cardiovascular Disease Risk? In Genetic Variation in Taste Sensitivity Edited by: Prescott J, Tepper BJ. Dekker; 2004

14. Basson MD, Bartoshuk LM, Dichello SZ, Panzini L, Weiffenbach JM, Duffy VB: Association between 6-n-propylthiouracil (PROP) bitterness and colonic neoplasms. Dig Dis Sci 2005 50(3):483-489.

15. Duffy VB, Davidson AC, Kidd JR, Kidd KK, Speed WC, Pakstis AJ, Reed DR, Snyder DJ, Bartoshuk LM: Bitter receptor gene (TAS2R38), 6-n-propylthiouracil (PROP) bitterness and alcohol intake. Alcohol Clin Exp Res 2004, 28(I I): 1629-1637.

16. Behrens M, Meyerhof W: Bitter taste receptors and human bitter taste perception. Cell Mol Life Sci 2006, 63(I3):I50I-I509.

17. Bufe B, Breslin PAS, Kuhn C, Reed DR, Tharp CD, Slack JP, Kim UK, Drayna $D$, Meyerhof $W$ : The molecular basis of individual differences in phenylthiocarbamide and propylthiouracil bitterness perception. Current Biology 2005, 15:322-327.

18. Bartoshuk LM, Davidson A, Kidd J, Kidd K, Speed W: Supertasting is not explained by the PTC/PROP gene. 2004

19. Bartoshuk LM, Duffy VB, Miller IJ: PTC/PROP tasting: anatomy, psychophysics, and sex effects. [erratum appears in Physiol Behav I995 Jul;58(I):203]. Physiology \& Behavior 1994, 56(6): $|165-| 17 \mid$.

20. White T, Longo M: Personailty traits and PROP sensitivity. 2004

21. Timpson NJ, Christensen CM, Lawlor DA, Gaunt TR, Day IN, Ebrahim S, Davey Smith G: TAS2R38 (PTC) haplotypes, coronary heart disease traits and eating behavior in the British Women's Heart and Health Study. American Journal of Clinical Nutrition 2005, 81: I005-101।.

22. Duffy VB, Bartoshuk LM: Food acceptance and genetic variation in taste. Journal of the American Dietetic Association 2000, 100(6):647-655

23. Miller IJ Jr.: Variation in human taste bud density as a function of age. Annals of the New York Academy of Sciences 1989, 561:307-319.
24. Miller IJ Jr.: Variation in human fungiform taste bud densities among regions and subjects. Anatomical Record 1986, 216(4):474-482.

25. Miller IJ Jr., Reedy FE Jr.: Variations in human taste bud density and taste intensity perception. Physiology \& Behavior 1990, 47(6): $1213-1219$

26. Delwiche JF, Buletic Z, Breslin PA: Relationship of papillae number to bitter intensity of quinine and PROP within and between individuals. Physiology \& Behavior 200I, 74(3):329-337.

27. Golding J, Pembrey M, Jones R, Team AS: ALSPAC--the Avon Longitudinal Study of Parents and Children. I. Study methodology. Paediatric and Perinatal Epidemiology 200I, I5(I):74-87.

28. The Avon Logitudinal Study of Parents and Children: website [http://www.alspac.bris.ac.uk]

29. Bartoshuk LM, Fast K, Snyder D]: Differences in our sensory worlds. Current Directions in Psychological Science 2005, I 4: I 22-125.

30. Jones RW, Ring S, Tyfield L, Hamvas R, Simmons H, Pembrey M, Golding J, Team AS: A new human genetic resource: a DNA bank established as part of the Avon longitudinal study of pregnancy and childhood (ALSPAC). European Journal of Human Genetics 2000, 8(9):653-660

31. KBioscience: Genotyping Chemistry [http://www.kbio science.co.uk/genotyping/genotyping-chemistry.htm]

32. KBiosciences: website [http://www.kbioscience.co.uk]

33. HMSO: Standard Occupational Classification. Office of Population Census and Surveys 1990.

34. Angold A, Costello EJ, Messer SC, Pickles A, Winder F, Silver D: Development of a short questionnaire for use in epidemiological studies of depression in children and adolscents. International Journal of Methods in Psychiatric Research 1995, 5:237-249.

35. Loeber R, Stouhamer-Loeber M, Van Kammen WB, Farrington DP Development of a new measure of self-reported antisocial behaviour for young children: prevalence and reliability. In Cross-national research in self-reported crime and delinquency Edited by: Klein MW. Kluwer Academic Publishers; 1989.

36. Stephens M, Donnelly P: A comparison of Bayesian methods for haplotype reconstruction from population genotype data. American Journal of Human Genetics 2003, 73: I 162-II69.

37. Guo SW, Thompson EA: Performing the exact test of HardyWeinberg proportion for multiple alleles. Biometrics 1992, 48(2):36I-372.

38. Breiman L: Statistics: with a view towards applications. Boston , Houghton Mifflin; 1973.
Publish with Biomed Central and every scientist can read your work free of charge

"BioMed Central will be the most significant development for disseminating the results of biomedical research in our lifetime. "

Sir Paul Nurse, Cancer Research UK

Your research papers will be:

- available free of charge to the entire biomedical community

- peer reviewed and published immediately upon acceptance

- cited in PubMed and archived on PubMed Central

- yours - you keep the copyright
BioMedcentral 